\title{
Comparison of predictive methods for flow boiling heat transfer in conventional channels and minichannels - the effect of reduced pressure
}

\author{
Dariusz Mikielewicz ${ }^{1}$, Blanka Jakubowska ${ }^{1, *}$ \\ ${ }^{1}$ Gdansk University of Technology, Faculty of Mechanical Engineering, Department of Energy and Industrial Apparatus, \\ Narutowicza 11/12, 80-233 Gdansk, Poland
}

\begin{abstract}
In the paper are presented the results of follow on studies from [1]-[3] using authors own model to predict heat transfer coefficient during flow boiling. The model has been tested against a large selection of experimental data collected from various researchers to investigate the sensitivity of the in-house developed model. The collected experimental data came from various studies from literature and were conducted for the full range of quality variation and a wide range of mass velocity and saturation temperatures. In the work are presented the results of calculations obtained using the in-house developed semi empirical model on selected experimental flow boiling data of the refrigerants: R134a, R1234yf, R600a, R290, NH3, CO2, R236fa, R245fa, R152a and HFE7000. In the present study the particular attention was focused on the influence of reduced pressure on the predictions of the theoretical model. The results of calculations were to test the sensitivity of the flow boiling model with respect to selection of the appropriate two-phase flow multiplier, which is one of the distinctive elements of the in-house model. The main purpose of this paper however is to show the effect of the reduced pressure on the predictions of heat transfer during flow boiling.
\end{abstract}

\section{Introduction}

Nowadays, there is an increasing interest in refrigerants featuring low Global Warming Potential (GWP). The reason of this concern can be attributed to the growing number of regulations and laws prohibiting the use of some of synthetic refrigerants [4]. According to this regulations the new fluids used in e.g. air-conditioning and refrigeration applications cannot be manufactured with fluorinated greenhouse gases having GWP greater than 150 [5]. Within the Montreal Protocol [5], most of the substances used in refrigeration system had been regulated due to its Ozone Depletion Potential (ODP). Consequently one of the working fluids most extensively used in medium evaporation temperatures, i.e. refrigerant R134a has a 100-year GWP=1430 [6], needs to be replaced by more environmentally friendly fluids. Previous studies have considered R152a [7] and the natural refrigerant $\mathrm{CO}_{2}$ [8] as possible replacements for R134a. However, R152a is a flammable refrigerant and for the $\mathrm{CO}_{2}$ system, the working pressure is significantly higher than for R134a system. Apart from that drawback $\mathrm{CO}_{2}$, as compared to other contemporary fluids is a relatively safe one. It is non-toxic, non-flammable, nonexplosive, cheap and can be coupled with most metals and plastics. Design of evaporators for use of the $\mathrm{CO}_{2}$ requires the exact determination of the heat transfer coefficient during flow boiling. Available in the literature empirical correlations give different results as compared to the results obtained experimentally. There is hardly any robust and recommended correlation for the purpose of calculation of carbon dioxide two-phase heat transfer, despite some devoted contributions [9]. Moreover, the traditional refrigerants can be also replaced by other natural refrigerants such as R600a and R290. These working fluids do not exhibit a harmful impact on ozone layer and have a negligible GWP. Furthermore, the mixture of R290 and R600a can be one of the most appropriate refrigerants to replace R134a [10]. Recently, the new fluid R1234yf has been believed to be the promising candidate as an alternative of R134a. Its $\mathrm{ODP}=0$, whereas $\mathrm{GWP}=4$ and its thermophysical properties are similar to those of R134a. There are many available experimental investigations into flow boiling heat transfer of all mentioned above working fluids in the literature. Unfortunately, the results published for some of these fluids are still inconsistent and difficult in mathematical modelling [3]. In the literature there are many empirical correlations for modelling of flow boiling heat transfer. As mentioned earlier, in case of $\mathrm{CO}_{2}$ most of well-known models does not exhibit a good consistency with experimental data. It was authors intention to show the performance of their own approach in predicting of flow boiling heat transfer coefficient on the example of the data collected from literature using the in-house developed model [11]-[14]. Based on the evidence of comparisons with experimental data a

\footnotetext{
Corresponding author: blanka.jakubowska@pg.edu.pl
} 
correction incorporating the effect of reduced pressure has been postulated to the authors own model to provide the best consistently of the predictions with the experimental data.

\section{The model}

The versatile semi-empirical model for calculations of flow boiling and flow condensation due to $\mathrm{J}$. Mikielewicz [11] and the final version due to $D$. Mikielewicz et al. [12]-[14] has been tested for a significant number of experimental data and has returned satisfactory results for the case of the flow boiling process for numerous fluids. The fundamental hypothesis of the model is the fact that heat transfer during flow boiling with bubble generation can be modeled as a sum of two contributions constituting the total energy dissipation in the flow, namely the energy dissipation due to the shearing flow without the bubbles and dissipation resulting from the bubble generation. The final version of the model reads:

$$
\frac{\alpha_{T P B}}{\alpha_{L O}}=\sqrt{R_{M S}^{n}+\frac{1}{1+P}\left(\frac{\alpha_{P b}}{\alpha_{L O}}\right)^{2}}
$$

Equation (1) also includes the empirical correction $P$ defined by equation (2). Occurring in the equation (1) the two-phase multiplier is raised to the power $n(n=0.76$ for turbulent flow, $\mathrm{n}=2$ for laminar flow).

$$
P=2.53 \cdot 10^{-3} \cdot \mathrm{Re}^{1.17} \cdot B o^{0.6} \cdot\left(R_{M S}-1\right)^{-0.65}
$$

In calculations tested was the sensitivity of the developed model to the selection of the two-phase flow multiplier. For that purpose four models were introduced into equation (1), namely modified correlation due to Muller-Steinhagen and Heck [16], Friedel [17], Tran [18] and taking into account of the non-adiabatic effect [14]. The relationships, which describes the two-phase multiplier using during this analyse has been show in Table 1.

\begin{tabular}{|c|c|}
\hline Autor(s) & Equation \\
\hline $\begin{array}{l}\text { Muller- } \\
\text { Steinhagen } \\
\text { and Heck } \\
{[16]}\end{array}$ & $\begin{array}{l}R_{M S}=\left[1+2 \cdot\left(\frac{1}{f_{1}}-1\right) \cdot x \cdot \operatorname{Con}^{m}\right] \cdot(1-x)^{\frac{1}{3}}+x^{3} \cdot \frac{1}{f_{1 z}} \\
\text { Turbulent flow } \\
f_{I}=\left(\rho_{L} / \rho_{G}\right)\left(\mu_{L} / \mu_{G}\right)^{0.25} \\
f_{1 z}=\left(\mu_{G} / \mu_{L}\right)\left(\lambda_{L} / \lambda_{G}\right)^{1.5}\left(c_{p L} / c_{p G}\right) \\
\text { Laminar flow } \\
f_{I}=\left(\rho_{L} / \rho_{G}\right)\left(\mu_{L} / \mu_{G}\right) \\
f_{I z}=\left(\lambda_{G} / \lambda_{L}\right) \\
m=0 \text { for flow in conventional channels } \\
m=-1 \text { for flow in minichannels. }\end{array}$ \\
\hline Friedel [17] & $\begin{array}{c}R_{F}=E+\frac{3.24 \cdot F \cdot H}{F r^{0.045} \cdot W e^{0.035}} \\
E=(1-x)^{2}+x^{2}\left(\frac{\rho_{l} f_{v}}{\rho_{v} f_{l}}\right) \\
F=x^{0.78}(1-x)^{0.2224} \\
H=\left(\frac{\rho_{l}}{\rho_{v}}\right)^{0.91}\left(\frac{\mu_{v}}{\mu_{l}}\right)^{0.19}\left(1-\frac{\mu_{v}}{\mu_{l}}\right)^{0.7}\end{array}$ \\
\hline
\end{tabular}

Table 1. Two-phase multiplier correlations

\begin{tabular}{|l|c|}
\hline Tran [18] & $R_{T}=1+\left[4,3 \cdot \frac{(d p / d z)_{G O}}{(d p / d z)_{L O}}\right] \times$ \\
& {$\left[N_{\text {conf }} \cdot x^{0.875} \cdot(1-x)^{0.875}+x^{1,75}\right]$} \\
$N_{\text {conf }}=\sqrt{\frac{\sigma}{g\left(\rho_{l}-\rho_{g}\right) \cdot d_{h}^{2}}}$ \\
\hline $\begin{array}{l}\text { non-adiabatic } \\
\text { effects [14] }\end{array}$ \\
$R_{B}=\left\{\begin{array}{l}R_{M S} \cdot\left(1-\frac{B}{2}\right) \quad f o r 0.1<x \leq 1 \\
R_{M S} \sqrt{1+\left(\frac{8 \cdot \alpha_{P b} \cdot d_{w}}{\lambda_{l} \cdot \operatorname{Re} \cdot \operatorname{Pr} \cdot \xi_{0} \cdot R_{M S}}\right)^{2}} \quad \text { for } 0 \leq x \leq 0.1 \\
B=\frac{2 \cdot q \cdot \frac{\rho_{l}}{\rho_{v}}}{f_{r} \cdot G(s-1) \cdot r}, \quad s=\sqrt[3]{\frac{\rho_{l}}{\rho_{v}}}\end{array}\right.$ \\
\hline
\end{tabular}

It was expected that the accuracy of model predictions could be improved by some modifications to the empirical correction $\mathrm{P}$, here by incorporation of the reduced pressure effect. The modified empirical correction $\mathrm{P}$ yields:

$$
P=2.53 \cdot 10^{-3} \cdot \operatorname{Re}^{1.17} \cdot B o^{0.6} \cdot\left(R_{M S}-1\right)^{-0.65} \cdot\left(\frac{p_{s a t}}{P_{k r}}\right)^{a}
$$

In the following part, the basic model and its subsequent modifications, which have been selected for discussion, will be analysed with respect to predictions of the heat transfer coefficient. These models are denoted respectively as: model I - using equation (3), model II - using equation (4), model III - using equation (5) and model IV - using equation (6). Moreover, exponent a, present in the modified two-phase flow multiplier in equation (7) was adjusted to the available data bank for flow boiling.

\section{Results and discussion}

The value of the confinement number Con and reduced pressure for all considered refrigerants are presented in Table 2. Using the Kew and Cornwell [19] criterion, the available data bank was divided into conventional size

\begin{tabular}{|c|c|c|c|}
\hline Author(s) & Fluid & Con & $P_{\text {sat }} / P_{k r}$ \\
\hline $\begin{array}{c}\text { Docoloumbier et } \\
\text { al. [20] }\end{array}$ & \multirow{5}{*}{$\mathrm{CO}_{2}$} & $\begin{array}{c}1.611- \\
1.412\end{array}$ & $\begin{array}{c}0.359 \\
- \\
0.472\end{array}$ \\
\hline Pamitran et al. [21] & & $\begin{array}{c}0.241- \\
0.483\end{array}$ & $\begin{array}{r}0.485 \\
-0.61\end{array}$ \\
\hline $\begin{array}{l}\text { Mastrullo et al. } \\
\qquad[22]\end{array}$ & & $\begin{array}{c}0.113- \\
0.138\end{array}$ & $\begin{array}{c}0.382 \\
- \\
0.548\end{array}$ \\
\hline Yoon et al. [23] & & $\begin{array}{l}0.083- \\
0.099\end{array}$ & $\begin{array}{r}0.472 \\
-0.61\end{array}$ \\
\hline Choi et al. [24] & & 0.415 & 0.61 \\
\hline
\end{tabular}
channels and minichannels.

Table 2. List of experimental data. 


\begin{tabular}{|c|c|c|c|}
\hline Oh et al. [25] & & $\begin{array}{c}0.071- \\
0.274\end{array}$ & $\begin{array}{l}0.413 \\
0.777\end{array}$ \\
\hline Dang et al. [26] & & 0.274 & 0.69 \\
\hline Kim et al. [27] & & $\begin{array}{c}0.096- \\
0.16\end{array}$ & $\begin{array}{c}0.413 \\
- \\
0.777\end{array}$ \\
\hline Wu et al. [28] & & $\begin{array}{c}0.526- \\
0.78\end{array}$ & $\begin{array}{c}0.136 \\
- \\
0.472\end{array}$ \\
\hline Cho et al. [29] & & $\begin{array}{c}0.048- \\
0.149\end{array}$ & $\begin{array}{c}0.472 \\
- \\
0.777\end{array}$ \\
\hline Zhao et al. [30] & & 0.223 & 0.19 \\
\hline Choi et al. [24] & \multirow[t]{2}{*}{$\mathrm{NH}_{3}$} & $\begin{array}{c}0.75- \\
1.5\end{array}$ & 0.0455 \\
\hline Anwar [31] & & 1.167 & 0.1125 \\
\hline Copetti et al. [32] & \multirow{2}{*}{ R600a } & 0.537 & 0.0884 \\
\hline Anwar [31] & & 0.855 & 0.1024 \\
\hline Choi et al. [24] & \multirow{3}{*}{ R290 } & $\begin{array}{l}0.5- \\
0.889\end{array}$ & $\begin{array}{c}0.111 \\
- \\
0.154\end{array}$ \\
\hline Wang et al. [33] & & $\begin{array}{l}0.08- \\
0.272\end{array}$ & $\begin{array}{c}0.0328 \\
- \\
0.105\end{array}$ \\
\hline Anwar [31] & & 0.021 & 0.273 \\
\hline Anwar [31] & $\mathrm{R} 152 \mathrm{a}$ & $\begin{array}{c}0.639- \\
0.656\end{array}$ & $\begin{array}{l}0.14- \\
0.162\end{array}$ \\
\hline Lu et al. [34] & \multirow{9}{*}{$\mathrm{R} 134 \mathrm{a}$} & 0.234 & 0.129 \\
\hline Satioh et al. [35] & & 0.444 & 0.151 \\
\hline Copetti et al. [32] & & 0.328 & 0.149 \\
\hline Kundu et al. [36] & & 0.132 & 0.094 \\
\hline Xu et al. [37], [38] & & $\begin{array}{c}0.205- \\
0.38\end{array}$ & $\begin{array}{c}0.142 \\
- \\
0.202\end{array}$ \\
\hline Mancin et al. [39] & & 0.238 & 0.189 \\
\hline $\begin{array}{c}\text { Tibirçá and } \\
\text { Ribatski [40] }\end{array}$ & & $\begin{array}{c}0.325- \\
0.371\end{array}$ & $\begin{array}{c}0.149 \\
- \\
0.257\end{array}$ \\
\hline Owhaib et al. [41] & & 0.645 & 0.213 \\
\hline Shiferaw et al. [42] & & 0.732 & 0.195 \\
\hline
\end{tabular}

\begin{tabular}{|c|c|c|c|}
\hline $\begin{array}{c}\text { Martin-Callizo et } \\
\text { al. [43] }\end{array}$ & & 1.224 & 0.218 \\
\hline $\begin{array}{c}\text { Consolini and } \\
\text { Thome [44] }\end{array}$ & & 1.579 & 0.195 \\
\hline $\begin{array}{l}\text { Mahmoud et al. } \\
\qquad 45]\end{array}$ & & $\begin{array}{c}0.731- \\
1.65\end{array}$ & $\begin{array}{c}0.145 \\
- \\
0.197\end{array}$ \\
\hline $\begin{array}{c}\text { Ong and Thome } \\
{[46]}\end{array}$ & & 0.782 & 0.195 \\
\hline Anwar et al. [47] & & $\begin{array}{c}0.499- \\
\mathbf{1 . 2 6 7}\end{array}$ & $\begin{array}{l}0.174 \\
-0.2\end{array}$ \\
\hline Del Col et al. [4] & \multirow{5}{*}{ R1234yf } & 0.736 & 0.238 \\
\hline Satioh et al. [35] & & 0.414 & 0.151 \\
\hline Choi et al. [24] & & $\begin{array}{c}0.285- \\
0.567\end{array}$ & $\begin{array}{c}0.111 \\
- \\
0.133\end{array}$ \\
\hline Diani et al. [48] & & 0.218 & 0.232 \\
\hline Anwar et al. [47] & & $\begin{array}{c}0.456- \\
0.475\end{array}$ & $\begin{array}{c}0.213 \\
- \\
0.244\end{array}$ \\
\hline $\begin{array}{c}\text { Ong and Thome } \\
{[46]}\end{array}$ & R236fa & 0.826 & 0.104 \\
\hline $\begin{array}{c}\text { Tibirçá and } \\
\text { Ribatski [40] }\end{array}$ & \multirow{3}{*}{$\mathrm{R} 245 \mathrm{fa}$} & $\begin{array}{l}0.441 \\
0.425\end{array}$ & $\begin{array}{l}0.050 \\
0.071\end{array}$ \\
\hline $\begin{array}{c}\text { Ong and Thome } \\
{[46]}\end{array}$ & & 0.986 & 0.050 \\
\hline Anwar [31] & & 1.592 & 0.049 \\
\hline $\begin{array}{c}\text { Own experimental } \\
\text { research }\end{array}$ & HFE700 & $\begin{array}{c}0,361- \\
0,396\end{array}$ & $\begin{array}{c}0.034 \\
- \\
0.080\end{array}$ \\
\hline
\end{tabular}

It can be concluded that the transition from conventional size channels to minichannels takes place on average at channel diameter smaller than $1.5 \mathrm{~mm}$, i.e. Con $>0.5$. It should be also noted that the analysis of the parameters from Table 2. indicates the fact that collected for scrutiny experimental research covers a full range of quality and a relatively wide range of mass velocity. Belyaev et al. [49] tried to confirm the hypothesis that in case of high value of reduced pressure, the two-phase flow structures in small diameter channels are similar to those occurring in conventional size diameter. Based on their [49] study they observed that when reduced pressure is greater than 0.4 then is no differences between heat transfer during flow boiling in minichannels and conventional channels. 
Figures 1 to 4 show the results of calculations of heat transfer coefficient for all data bank obtained using the described earlier flow boiling model described by Eq. (1), (2) and (6), supplemented by different definitions of the two-phase flow multiplier (3), (4) and (5).

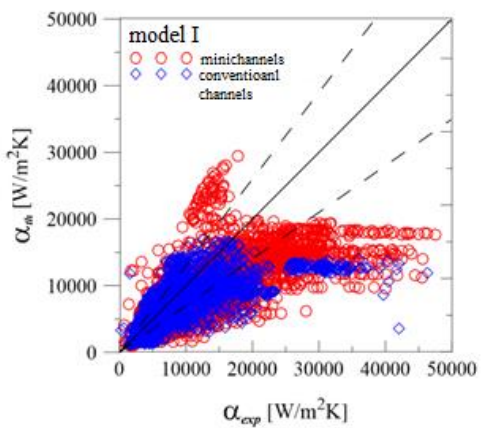

Fig. 1. Comparison of the test results $\alpha \exp$ with predictions $\alpha$ th using Muller Steinhagen and Heck model, Eq. (3).

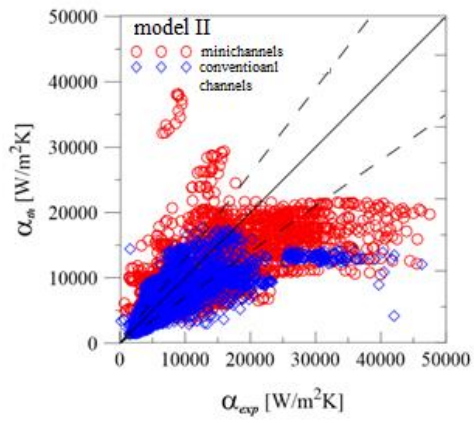

Fig. 2. Comparison of the test results $\alpha_{\exp }$ with predictions $\alpha_{t h}$ using Friedel model Eq. (4).

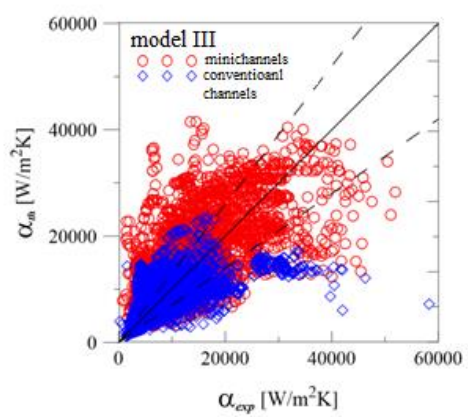

Fig. 3. Comparison of the test results $\alpha_{\exp }$ with predictions $\alpha_{\mathrm{th}}$ using Tran et al. model Eq. (5).

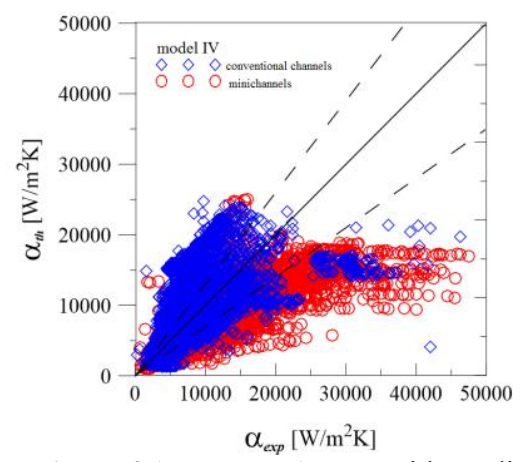

Fig. 4. Comparison of the test results $\alpha_{\exp }$ with predictions $\alpha_{\mathrm{th}}$ using Eq. (6).

The modification to the empirical correction described by Eq. (2) includes the effect of reduced pressure. The new version of the correction $P$ is presented by Eq. (7), where the reduced pressure is raised to power $a$. The value of the exponent $a$ was adjusted using the regression analysis. The results of calculations, which were obtained with taking into account of the reduced pressure are presented in Figures 5 to 8 . The information about approximating error $A E$ and correlation factors $R^{2}$ is given in Table 3 .

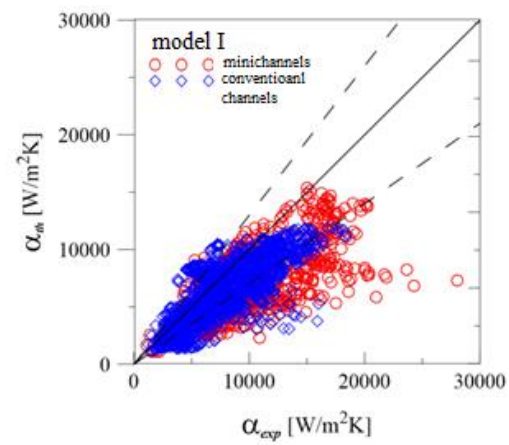

Fig. 5. Comparison of the test results $\alpha_{\exp }$ with predictions $\alpha_{t h}$ using Eqs. (3), (7) and $a=1$ for synthetic refrigerants: R134a, R1234yf, R236fa, R245fa, R152a, HFE7000.

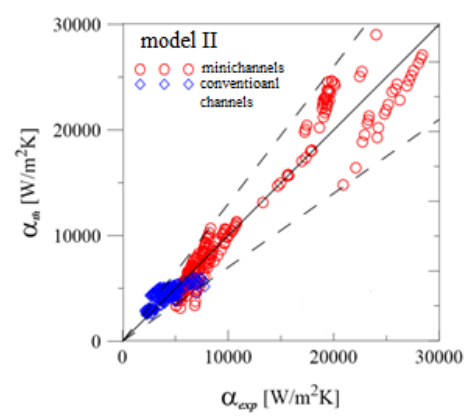

Fig. 6. Comparison of the test results $\alpha_{\exp }$ with predictions $\alpha_{t h}$ using Eqs. (4), (7) and $a=-0.86$ for natural refrigerants: R600a, $\mathrm{NH}_{3}, \mathrm{R} 290$

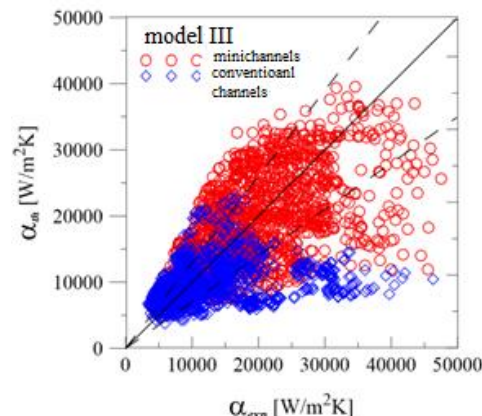

Fig. 7. Comparison of the test results $\alpha_{\exp }$ with predictions $\alpha_{t h}$ using Eqs. (5), (7) and $a=1$ for $\mathrm{CO}_{2}$.

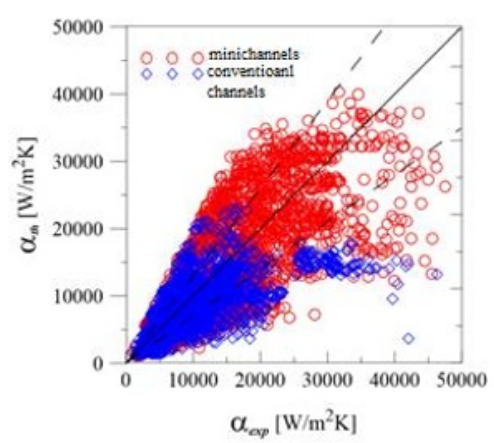

Fig. 8. Comparison of the test results $\alpha_{\exp }$ with predictions $\alpha_{t h}$ for final version of correlation. 
Table 3. Values of exponent $a, A E$ and $R^{2}$.

\begin{tabular}{|c|c|c|c|c|}
\hline Model & $a$ & data & $\boldsymbol{A E}[\%]$ & $R^{2}$ \\
\hline \multirow{4}{*}{ Model I } & \multirow{16}{*}{0} & all & 33.11 & 0.426 \\
\hline & & natural & 37.41 & 0.548 \\
\hline & & synthetic & 30.20 & 0.239 \\
\hline & & $\mathrm{CO}_{2}$ & 38.46 & 0.243 \\
\hline \multirow{4}{*}{ Model II } & & all & 32.64 & 0.344 \\
\hline & & natural & 33.45 & 0.344 \\
\hline & & synthetic & 24.63 & 0.184 \\
\hline & & $\mathrm{CO}_{2}$ & 34.18 & 0.297 \\
\hline \multirow{4}{*}{ Model III } & & all & 50.17 & 0.115 \\
\hline & & natural & 30.34 & 0.142 \\
\hline & & synthetic & 52.10 & 0.050 \\
\hline & & $\mathrm{CO}_{2}$ & 31.25 & 0.349 \\
\hline \multirow{4}{*}{ Model IV } & & all & 44.72 & 0.221 \\
\hline & & natural & 46.50 & 0.483 \\
\hline & & synthetic & 49.00 & 0.072 \\
\hline & & $\mathrm{CO}_{2}$ & 40.34 & 0.216 \\
\hline \multirow{3}{*}{ Model I } & -0.86 & natural & 32.40 & 0.513 \\
\hline & 1 & synthetic & 27.62 & 0.275 \\
\hline & 1 & $\mathrm{CO}_{2}$ & 37.74 & 0.256 \\
\hline \multirow{3}{*}{ Model II } & -0.86 & natural & 28.60 & 0.338 \\
\hline & 1 & synthetic & 23.36 & 0.250 \\
\hline & 1 & $\mathrm{CO}_{2}$ & 33.84 & 0.320 \\
\hline \multirow{3}{*}{ Model III } & -0.86 & natural & 25.88 & 0.150 \\
\hline & 1 & synthetic & 24.33 & 0.201 \\
\hline & 1 & $\mathrm{CO}_{2}$ & 31.08 & 0.362 \\
\hline \multirow{3}{*}{ Model IV } & -0.86 & natural & 43.34 & 0.415 \\
\hline & 1 & synthetic & 47.46 & 0.090 \\
\hline & 1 & $\mathrm{CO}_{2}$ & 41.00 & 0.196 \\
\hline \multicolumn{3}{|c|}{ All data presented in Figure 8} & 28.00 & 0.490 \\
\hline
\end{tabular}

\section{Conclusions}

The paper presents the analysis of the results of flow boiling calculations using an authors' own model to predict heat transfer coefficient. The model was studied in several way, i.e., it was used as original one, in a modified version where the two-phase flow multiplier was changed and also in modified version where into the empirical correction $P$ was included the reduced pressure effect (the value of exponent a was modeled). The results show that change the model which describing two-phase multiplier is significant especially in case of $\mathrm{CO}^{2}$, where the best compliance with experimental data obtained using Tran correlation. The results also show that taking into account appropriate two-phase multiplier model and reduced pressure effects can significantly contribute to the convergence with experimental data compared to original model. In authors' opinion, the proposed method to calculate the heat transfer coefficient is a reliable tool in engineering calculations e.g. designing heat exchangers $[50,51]$.

\section{Acknowledgements}

The results presented in the paper have been partially funded from the project 2017/25/B/ST8/00755 by the National Science Centre, Poland.

\section{References}

1 D. Mikielewicz, B. Jakubowska, Arch Thermodyn., 35, 97-114 (2014)

2 D. Mikielewicz, B. Jakubowska, Arch. Thermodyn., 37, 89-106 (2016)

3 D. Mikielewicz, B. Jakubowska, Polish Marit. Res., 24, 141-148 (2017)

4 D. Del Col, S. Bortolini, D. Torresin, A. Cavallini, Procedings of 23rd IIR International Congress of Refrigeration, Prague, Czech Republic (2011)

5 United Nations Environment Program (UNEP) Montreal Protocol on Substances that Deplete the Ozone Layer, Final Act. United Nations, New York, USA, (1997)

6 M.P. Simmonds, W. J. Elliott, J. Mar. Biol. Assoc. UK, 89, 203-210 (2009)

7 M. Ghodbane, Int. Proceedings of the international congress and exposition, Detroit, USA (1999)

8 G. Lorentzen, Revival of carbon dioxide as refrigerant, Int. J. Refrig., 17, 292-301 (1994)

9 L. Cheng, G. Ribatski, J. R. Thome, Int. J. Heat Mass Transf. 51, 125-135 (2008)

10 S. Wongwiesess, N. Chimres, Energ. Convers. Manag., 46, 85-100 (2005)

11 J. Mikielewicz, Int. J. Heat Transf., 17, 1129-1134 (1973)

12 D. Mikielewicz, J. Mikielewicz, J. Tesmar, Int. J. Heat Mass Transf., 50, 3949-3956 (2007)

13 D. Mikielewicz, J. Mikielewicz, Heat Transf. Eng., 32, 1173-1181 (2011)

14 D. Mikielewicz, R. Andrzejczyk, B. Jakubowska, J. Mikielewicz, Heat Transf. Eng., 37, 1158-1171 (2016)

15 H. Müller-Steinhagen, K. Heck, Chem. Eng. Prcoess, 20, 197-308 (1986)

16 D. Mikielewicz, Heat Transf. Eng., 31, 276-287 (2010)

17 L. Friedel, European Two-Phase Flow Group Meeting, Paper E2, Ispra, Italy (1979)

18 T. N. Tran, M. C. Chyu, M. W. Wambsganss, Int. J. Refrig., 26, 1739-1754 (2000)

19 P. A. Kew, K. Cornwell, Appl. Therm. Eng., 17, 705-715 (1997)

20 M. Docoulombier, S. Colasson, B. J. P. Haberschil, Exp. Therm. Fluid Sci., 35, 597 - 611 (2011)

21 A. S. Pamitran, K.-I. Choi, J.-T. Oh, Nasruddin, Int. Multiph. Flow, 37, 794-801 (2011)

22 R. Mastrullo, A. W. Mauro, A. Rosato, G. P. Vanoli, Int. J. Refrig., 33, 1068-1085 (2010) 
23 S. H. Yoon, E. S. Cho, Y. W. Hwang, M. S. Kim, K. Min, Y. Kim, Int. J. Refrig, 27, 111-119 (2004)

24 K.-I. Choi, A. S. Pamitran, C.-Y. Oh, J.-T. Oh, Int. J. Refrig., 30, 1336-1346 (2007)

25 H.-K. Oh, C. H. Son, Appl. Therm. Eng., 31, 163$172(2011)$

26 C. Dang, N. Haraguchi, E. Hihara, Int. J. Refrig, 33, 655-663 (2010)

27 Y. J. Kim, J. M. Cho, M. . Kim, Int. J. Refrig., 31, 771-779 (2008)

28 J. Wu, T. Koettig, C. Franke, D. Helmer, T. Eisel, F. Haug, J. Bremer, Int. J. Heat Mass Transf., 54, 2154-2162 (2011)

29 J. M. Cho, M. S. Kim, Int. J. Refrig., 30, 986-994 (2007)

30 X. Zhao, P. K. Bansal, Int. J. Refrig., 30, 937-945 (2007)

31 Z. Anwar, Flow boiling heat transfer, pressure drop and dryout characteristics of low GWP refrigerants in a vertical mini-channel, Stockholm, Sweden (2014)

32 J. B. Copetti, M. H. Macaganan, F. Zinani, Int. J. Refrig., 33, 325-334 (2013)

33 C. C. Wang, C. S. Chiang, D. C. Lu, Exp Therm. Fluid Sci, 15, 395-405 (1997)

34 M.-C. Lu, J.-R. Tong, W.C-C, Int. J. Heat Mass Transf., 65, 545-551 (2013)

35 S. Satioh, C. Dang, Y. Nakamura, E. Hihara, Int. J. Refrig., 33, 1846-1853 (2011)

36 A. Kundu, R. Kumar, A. Gupta, Exp. Therm. Fluid Sci., 57, 344-352 (2014)

37 Y. Xu, X. Fang, G. Li, D. Li, Int. J. Heat Mass Transf., 80, 597-606 (2015)

38 Y. Xu, X. Fang, G. Li, D. Li, Int. J. Heat Mass Transf., 92, 1143-1157 (2016)

39 S. Mancin, A. Diani, L. Rossetto, Energy Procedia, 45, 6098-615 (2014)

40 C. B. Tibirçá, G. Ribatski, Int. J. Heat Mass Transf., 53, 2459-2468 (2010)

41 W. Owhaib, Experimental Heat Transfer, Pressure Drop and Flow Visualization of R-134a in Vertical Mini/Micro Tubes, Stockholm, Sweden (2007)

42 D. Shiferaw, T. G. Karayiannis, D. B. R. Kenning, Int. J Therm. Sci., 48, 331-341 (2009)

43 C. Martin-Callizo, R. Ali, B. Palm, Heat Transfer Conference Proceedings, Edinburgh, UK (2007)

44 L. Consolini, J. R. Thome, Microfluid Nanofluid, 6, 731-746 (2009)

45 M. M. Mahmoud, D. B. R. Kenning, T. G. Karayiannis, 7th Int. Conference in Enhanced, Compact and Ultra-compact Heat Exchangers: From Microscale Phenomena to Industrial Applications, Heredia, Costa Rice (2009)

46 C. I. Ong, J. R. Thome, Exp. Therm. Fluid Sci., 33, 651-663 (2009)

47 Z. Anwar, B. Palm, R. Khodabanden, Exp. Therm. Fluid Sci., 66, 137-149 ( 2015)

48 A. Diani, S. Mancin, L. Rossetto, Exp. Therm. Fluid Sci., 66, 127-136 (2015)

49 A. V Belyaev, A. N. Varava, A. V Dedov, A. T. Komov, Int. J. Heat Mass Transf., 110 360-373 (2017)
50 R. Andrzejczyk, T. Muszyński, App. Therm. Eng., 136, 237-251 (2018)

51 J. Wajs, D. Mikielewicz, B. Jakubowska, Energy, 157, 853-861 (2018) 